\title{
The Existence of Solution of a Critical Fractional Equation
}

\author{
Hui Chen \\ College of Science, University of Shanghai for Science and Technology, Shanghai, China \\ Email: 18721287390@163.com
}

How to cite this paper: Chen, H. (2019) The Existence of Solution of a Critical Fractional Equation. Journal of Applied Mathematics and Physics, 7, 243-253. https://doi.org/10.4236/jamp.2019.71020

Received: January 4, 2019

Accepted: January 28, 2019

Published: January 31, 2019

Copyright (C) 2019 by author(s) and Scientific Research Publishing Inc. This work is licensed under the Creative Commons Attribution International License (CC BY 4.0).

http://creativecommons.org/licenses/by/4.0/

\begin{abstract}
In this paper, we study the existence of solution of a critical fractional equation; we will use a variational approach to find the solution. Firstly, we will find a suitable functional to our problem; next, by using the classical concept and properties of the genus, we construct a mini-max class of critical points.
\end{abstract}

\section{Keywords}

Variational Approach, Fractional Laplacian, Minimax Principle, Genus

\section{Introduction}

In this paper, we focus our attention on the following problem:

$$
\begin{cases}(-\Delta)^{s} u=\lambda V(x)|u|^{p-1}+\beta K(x)|u|^{2_{s}^{*}-1} & \text { in } \Omega \\ u=0 & \text { in } R^{n} \backslash \Omega\end{cases}
$$

where $\Omega$ is a bounded domain in $R^{n}, \lambda>0,0<s<1$ and $n>2 s$, $1<p<2_{s}^{*}, \quad K(x) \in C\left(R^{n}\right) \cap L^{\infty}\left(R^{n}\right), \quad V(x) \geq 0$ and $V(x) \in C\left(R^{n}\right) \cap L^{q}\left(R^{n}\right)$ with $q=\frac{2_{s}^{*}}{2_{s}^{*}-p}$ here $(-\Delta)^{s}$ denotes the fractional Laplace operator defined, up to a normalization factor, by

$$
(-\Delta)^{s} u(x)=\int_{R^{n}} \frac{u(x)-u(y)}{|x-y|^{n+2 s}} \mathrm{~d} y, x \in R^{n} .
$$

The aim of this paper is to study the existence of solutions, we will see that if $1<p<2$, then by concentration-compactness principle, together with minimax arguments, we can prove the existence of solutions for (1.1). We now summarize the main result of the paper. 
Theorem 1.1. Let $1<p<2, K(x) \in C\left(R^{n}\right) \cap L^{\infty}\left(R^{n}\right)$ and $0 \leq V(x) \in C\left(R^{n}\right) \cap L^{q}\left(R^{n}\right)$ with $q=\frac{2_{s}^{*}}{2_{s}^{*}-p}$. Moreover, $V(x)>0$ is bounded on $\Omega$. Then

1) For any $\lambda>0$, there exists $\tilde{\beta}>0$, then for any $0<\beta<\tilde{\beta}$, (1.1) has a consequence of weak solutions $\left\{u_{n}\right\}$.

2) For any $\beta>0$, there exist $\tilde{\lambda}>0$, then for any $0<\lambda<\tilde{\lambda}$, (1.1) has a consequence of weak solutions $\left\{u_{n}\right\}$.

We denote by $H^{s}\left(R^{n}\right)$ the usual fractional Sobolev space endowed with the so-called Gagliardo norm

$$
\|u\|_{H^{s}\left(R^{n}\right)}=\|u\|_{L^{2}\left(R^{n}\right)}+\left(\int_{R^{n} \times R^{n}} \frac{|u(x)-u(y)|^{2}}{|x-y|^{n+2 s}} \mathrm{~d} x \mathrm{~d} y\right)^{\frac{1}{2}},
$$

Then we defined

$$
X_{0}^{s}(\Omega)=\left\{u \in H^{s}\left(R^{n}\right): u=0 \text { a.e. in } R^{n} \backslash \Omega\right\}
$$

endowed with the norm

$$
\|u\|_{X_{0}^{s}(\Omega)}=\left(\int_{R^{n} \times R^{n}} \frac{|u(x)-u(y)|^{2}}{|x-y|^{n+2 s}} \mathrm{~d} x \mathrm{~d} y\right)^{\frac{1}{2}},
$$

we refer to [1] for a general definition of $X_{0}^{s}(\Omega)$ and its properties.

Observe that by [[2], Proposition 3.6] we have the following identity

$$
\|u\|_{X_{0}^{s}(\Omega)}=\left\|(-\Delta)^{\frac{s}{2}} u\right\|_{L^{2}\left(R^{n}\right)} .
$$

In this work, the Sobolev constant is given by (can be seen in [[3], theorem 7.58])

$$
S(n, s):=\inf _{u \in H^{s}\left(R^{n}\right)\{\{0\}} Q_{n, s}(u)>0,
$$

where

$$
Q_{n, s}(u):=\frac{\int_{R^{n} \times R^{n}} \frac{\left|u(x)-u(y)^{2}\right|}{|x-y|^{n+2 s}} \mathrm{~d} x \mathrm{~d} y}{\left(\int_{R^{n} \times R^{n}}|u(x)|^{2_{s}^{*}} \mathrm{~d} x\right)^{\frac{2}{2_{s}^{*}}}}, u \in H^{s}\left(R^{n}\right)
$$

\section{Statements of the Result}

We will use a variational approach to find a solution of (1.1). Firstly, we will associate a suitable functional to our problem, the Euler-Lagrange functional related to problem (1) is given by $J: X_{0}^{s}(\Omega) \rightarrow R$ defined as follow

$$
J\left(u_{n}\right)=\frac{1}{2}\left\|u_{n}\right\|_{X_{0}^{s}(\Omega)}^{2}-\frac{\lambda}{p} \int_{\Omega} V(x)\left|u_{n}\right|^{p} \mathrm{~d} x-\frac{\beta}{2_{s}^{*}} \int_{\Omega} K(x)\left|u_{n}\right|^{2_{s}^{*}} \mathrm{~d} x .
$$


To proof that $J$ satisfy the Palais Smale condition at level $c$, we need the following lemma.

Lemma 2.1 [4] Letting $\phi$ be a regular function that satisfies that for some $\tilde{c}>0$

$$
|\phi(x)| \leq \frac{\tilde{c}}{1+|x|^{n+s}}, \quad x \in R^{n}
$$

and

$$
|\nabla \phi(x)| \leq \frac{\tilde{c}}{1+|x|^{n+s}}, \quad x \in R^{n}
$$

Let $B: X_{0}^{\frac{s}{2}}(\Omega) \times X_{0}^{\frac{s}{2}}(\Omega) \rightarrow R$ be a bilinear form defined by

$$
B(f, g)(x):=2 \int_{R} \frac{(f(x)-f(y))(g(x)-g(y))}{|x-y|^{n+s}} \mathrm{~d} y .
$$

then, for every $s \in(0,1)$, there exist positive constant $c_{1}$ and $c_{2}$, such that for $x \in R^{n}$, one has

$$
\left|(-\Delta)^{\frac{s}{2}} \phi(x)\right| \leq \frac{c}{1+|x|^{n+s}} \text { and }|B(\phi, \phi)(x)| \leq \frac{c}{1+|x|^{n+s}} .
$$

To establish the next auxiliary result we consider a radial, nonincreasing cut-off function

$$
\phi \in C_{0}^{\infty}\left(R^{n}\right) \text { and } \phi_{\varepsilon}(x):=\phi\left(\frac{x}{\varepsilon}\right)
$$

Lemma 2.2. [4] Letting $\left\{u_{m}\right\}$ be a uniformly bounded in $X_{0}^{s}(\Omega)$ and $\phi_{\varepsilon} \in C_{0}^{\infty}\left(R^{n}\right)$ the function defined in (2.6). Then,

$$
\lim _{\varepsilon \rightarrow 0} \lim _{m \rightarrow 0}\left|\int_{R^{n}} u_{m}(x)(-\Delta)^{\frac{s}{2}} \phi_{\varepsilon}(x)(-\Delta)^{\frac{s}{2}} u_{m}(x) \mathrm{d} x\right|=0 .
$$

Lemma 2.3. [4] With the same assumptions of Lemma 2.8 we have that

$$
\lim _{\varepsilon \rightarrow 0} \lim _{m \rightarrow 0}\left|\int_{R^{n}}(-\Delta)^{\frac{s}{2}} u_{m}(x) \mathrm{d} x B\left(u_{m}, \phi_{\varepsilon}\right)(x)\right|=0 .
$$

where $B$ is defined in (2.4).

Lemma 2.4. [5] (Minimax principle) Assume that $E \in C(X, \mathbb{R})$, and $\mathcal{A}$ is a family of nonempty subset of $X$, denote

$$
c=\inf _{A \in \mathcal{A}} \sup _{x \in A} E(x)
$$

If the following conditions holds:

1) $c$ is a finite real number;

2) there exists an $\bar{\varepsilon}>0$, such that $\mathcal{A}$ is invariant with respect to the family of mappings;

$$
\mathcal{T}=\{T \in(X, X) \mid T(x)=x, \text { if } E(x)<c-\bar{\varepsilon}\},
$$


that is, for any $T \in \mathcal{T}$, there holds

$$
A \in \mathcal{A} \Rightarrow \mathcal{T}(A) \in \mathcal{A}
$$

Then, $E$ possesses a $(P S)_{c}$ sequence at level $c$ define as (6.1.1); Furthermore, if $E$ satisfies the $(P S)_{c}$ condition (or the $(P S)_{c}$ condition at level $c$ ), then $c$ is a critical value of $E$.

\section{Proof of Theorem 1.1}

Firstly, recalling that $J$ is said to satisfy the Palais Smale condition at level $c$ if any sequence $\left\{u_{n}\right\} \in X_{0}^{s}(\Omega)$ such that $J\left(u_{n}\right) \rightarrow c$ and $J^{\prime}(u) \rightarrow 0$ has a convergent subsequence.

Lemma 3.1. The $(P S)_{c}$ sequence $\left\{u_{n}\right\}$ for $J$ is bounded.

Proof. Note that $\left\{u_{n}\right\} \subset X_{0}^{s}(\Omega)$ satisfies

$$
\begin{aligned}
J\left(u_{n}\right) & =\frac{1}{2}\left\|u_{n}\right\|_{X_{0}^{s}(\Omega)}^{2}-\frac{\lambda}{p} \int_{\Omega} V(x)\left|u_{n}\right|^{p} \mathrm{~d} x-\frac{\beta}{2_{s}^{*}} \int_{\Omega} K(x)\left|u_{n}\right|^{2_{s}^{*}} \mathrm{~d} x \\
& =c+o_{n}(1)
\end{aligned}
$$

and

$$
\begin{aligned}
\left\langle J^{\prime}\left(u_{n}\right), \phi\right\rangle & =\int_{\Omega}(-\Delta)^{s} u_{n} \mathrm{~d} x-\lambda \int_{\Omega} V(x)\left|u_{n}\right|^{p-2} u \phi \mathrm{d} x-\beta \int_{\Omega} K(x)\left|u_{n}\right|^{2_{s}^{*}-2} u \phi \mathrm{d} x \\
& =o_{n}(1)\|\phi\|_{X_{0}^{s}(\Omega)}, \quad \forall \phi \in X_{0}^{s}(\Omega)
\end{aligned}
$$

where $o_{n}(1) \rightarrow 0$ as $n \rightarrow \infty$. Choose $\phi=u_{n} \in X_{0}^{s}(\Omega)$ as test function in (3.2), we get that

$$
\begin{aligned}
o_{n}(1)\left\|u_{n}\right\|_{X_{0}^{s}(\Omega)} & =\left\langle J^{\prime}\left(u_{n}\right), u_{n}\right\rangle \\
& =\left\|u_{n}\right\|_{X_{0}^{s}(\Omega)}^{2}-\lambda \int_{\Omega} V(x)\left|u_{n}\right|^{p} \mathrm{~d} x-\beta \int_{\Omega} K(x)\left|u_{n}\right|^{2_{s}^{*}} \mathrm{~d} x \\
& =c+o_{n}(1) .
\end{aligned}
$$

therefore, by (3.1) and (3.2), we have

$$
\begin{aligned}
c & +o_{n}(1)-\frac{1}{2_{s}^{*}} o_{n}(1)\left\|u_{n}\right\|_{X_{0}^{s}(\Omega)} \\
= & \frac{1}{2}\left\|u_{n}\right\|_{X_{0}^{s}(\Omega)}^{2}-\frac{\lambda}{p} \int_{\Omega} V(x)\left|u_{n}\right|^{p} \mathrm{~d} x-\frac{\beta}{2_{s}^{*}} \int_{\Omega} K(x)\left|u_{n}\right|^{2_{s}^{*}} \mathrm{~d} x \\
& -\frac{1}{2_{s}^{*}}\left\|u_{n}\right\|_{X_{0}^{s}(\Omega)}^{2}-\frac{\lambda}{2_{s}^{*}} \int V(x)\left|u_{n}\right|^{p} \mathrm{~d} x-\frac{\beta}{2_{s}^{*}} \int_{\Omega} K(x)\left|u_{n}\right|^{2_{s}^{*}} \mathrm{~d} x \\
\geq & \frac{s}{n}\left\|u_{n}\right\|_{X_{0}^{s}(\Omega)}^{2}-\left(\frac{\lambda}{p}-\frac{1}{2_{s}^{*}}\right)\|V(x)\|_{L^{q}(\Omega)}\left\|u_{n}\right\|_{L^{2_{s}^{*}}}^{p} \\
\geq & \frac{s}{n}\left\|u_{n}\right\|_{X_{0}^{s}(\Omega)}^{2}-\left(\frac{\lambda}{p}-\frac{1}{2_{s}^{*}}\right) S(n, s)^{-\frac{p}{2}}\|V(x)\|_{L^{q}(\Omega)}\left\|u_{n}\right\|_{X_{0}^{s}(\Omega)}^{p} .
\end{aligned}
$$

which yields the boundeness of $\left\{u_{n}\right\}$ in $X_{0}^{s}(\Omega)$, since $1<p<2$.

If $K(x) \in L^{\infty}\left(\mathbb{R}^{n}\right)$, then for $2<p<2_{s}^{*}$, similar to the proof of $1<p<2$, we get 


$$
c+o_{n}(1)+o_{n}(1)\left\|u_{n}\right\|_{X_{0}^{s}(\Omega)} \geq\left(\frac{p-2}{2 p}\right)\left\|u_{n}\right\|_{X_{0}^{s}(\Omega)}^{2}-\frac{\left(p-2_{s}^{*}\right) \beta}{2_{s}^{*}} S^{-\frac{2_{s}^{*}}{2}}\left\|u_{n}\right\|_{X_{0}^{s}(\Omega)}^{2_{s}^{*}}
$$

Which also yields the boundedness of $(P S)_{c}$ sequence $\left\{u_{n}\right\}$.

Lemma 3.2. Assume that $c<0$. Then

1) For any $\lambda>0$, there exists $\beta_{0}>0$, such that for any $0<\beta<\beta_{0}$, then $J$ satisfies $(P S)_{c}$.

2) For any $\beta>0$ there exists $\lambda_{0}>0$ such that for any $0<\lambda<\lambda_{0}$, then $J$ satisfies $(P S)_{c}$.

Proof. By Lemma3.1 $\left\{u_{n}\right\}$ is bounded in $X_{0}^{s}(\Omega)$, up to a subsequence, we get that

$$
\begin{array}{ll}
u_{n} \rightarrow u & x \in X_{0}^{s}(\Omega) . \\
u_{n} \rightarrow u & x \in L^{r}(\Omega), 1 \leq r<2_{s}^{*} . \\
u_{n} \rightarrow u & \text { a.e. } x \in \Omega .
\end{array}
$$

Following [6] it is easy to prove that $X_{0}^{s}(\Omega)$ could also be the $X_{0}^{s}(\Omega)$ norm. Applying [[7], Theorem1.5], we have that the exist an index. Set $I \subseteq N$ a sequence of point $\left\{x_{k}\right\}_{x \in I \subset \Omega}$ and two sequences of nonnegative real numbers $\left\{\mu_{k}\right\}_{k \in I},\left\{v_{k}\right\}_{k \in I}$, such that

$$
\left|(-\Delta)^{\frac{s}{2}} u_{n}\right|^{2} \rightarrow \mu\left|(-\Delta)^{\frac{s}{2}} u\right|^{2}+\sum_{k \in I} \mu_{k} \delta_{x_{k}} .
$$

moreover

$$
\left|u_{n}\right|^{2_{s}^{*}} \rightarrow \mu|u|^{2_{s}^{*}}+\sum_{k \in I} v_{k} \delta_{x_{k}} .
$$

in the sense of measures, with

$$
v_{k} \leq S(s, n)^{-\frac{2_{s}^{*}}{2}} \mu_{k}^{\frac{2_{s}^{*}}{2}} \text { for every } k \in I
$$

here $\delta_{x_{k}}$ denotes the Dirac Delta at $x_{k}$, while $S(n, s)$ is the constant given in (1.7), we consider $\phi \in C_{0}^{\infty}\left(R^{n}\right)$ a nonincreasing cut-off function satisfying

$$
\phi=1 \text { in } B_{1}\left(x_{k_{0}}\right) \text { and } \phi=0 \text { in } B_{2}\left(x_{k_{0}}\right)^{c}
$$

Set $\phi_{\varepsilon}(x)=\phi\left(\frac{x}{\varepsilon}\right), x \in R^{n}$ taking the derivative of (1.6), for any $u, \phi \in X_{0}^{s}(\Omega)$. We obtain that

$$
\int_{R^{n} \times R^{n}} \frac{(u(x)-u(y))(\phi(x)-\phi(y))}{|x-y|^{n+2 s}} \mathrm{~d} x \mathrm{~d} y=\int_{R^{n}} \phi(x)(-\Delta)^{s} u(x) \mathrm{d} x
$$

Then, taking $\phi_{\varepsilon} u_{n}$ as a test function in $J^{\prime}\left(u_{n}\right) \rightarrow 0$

$$
\lim _{n \rightarrow 0} \int_{\mathbb{R}^{n}} \phi_{\varepsilon} u_{n}(-\Delta) u_{n} \mathrm{~d} x-\left(\lambda \int_{B_{2 \varepsilon}\left(x_{k_{0}}\right)} V(x) u_{n}^{p} \phi_{\varepsilon} \mathrm{d} x+\beta \int_{B_{2 \varepsilon}\left(x_{k_{0}}\right)} K(x) u_{n}^{2_{s}^{*}} \phi_{\varepsilon} \mathrm{d} x\right)=0
$$

by (3.10), we have 


$$
\begin{aligned}
& \lim _{n \rightarrow \infty} \int_{\mathbb{R}^{n}} u_{n}(x)(-\Delta)^{\frac{s}{2}} u_{n}(x)(-\Delta)^{\frac{s}{2}} \phi_{\varepsilon}(x) \mathrm{d} x \\
& -2 \int_{\mathbb{R}^{n}}(-\Delta)^{\frac{s}{2}} u_{n}(x) \int_{\mathbb{R}^{n}} \frac{\left(\phi_{\varepsilon}(x)-\phi_{\varepsilon}(y)\right)\left(u_{n}(x)-u_{n}(y)\right)}{|x-y|^{n+s}} \mathrm{~d} x \mathrm{~d} y \\
& =\lim _{n \rightarrow \infty} \lambda \int_{B_{2 \varepsilon}\left(x_{k_{0}}\right)} V(x)\left|u_{n}\right|^{p}(x) \phi_{\varepsilon}(x) \mathrm{d} x+\beta \int_{B_{2 \varepsilon}\left(x_{k_{0}}\right)} K(x)\left|u_{n}\right|^{2_{s}^{*}}(x) \phi_{\varepsilon}(x) \mathrm{d} x \\
& -\int_{B_{2 \varepsilon}\left(x_{k_{0}}\right)}\left((-\Delta)^{\frac{s}{2}} u_{n}\right)^{2} \phi_{\varepsilon}(x) \mathrm{d} x .
\end{aligned}
$$

therefore, by (3.5) (3.6) and (3.7) we get

$$
\begin{aligned}
& \lim _{\varepsilon \rightarrow 0} \lim _{n \rightarrow \infty} \int_{\mathbb{R}^{n}} u_{n}(x)(-\Delta)^{\frac{s}{2}} u_{n}(x)(-\Delta)^{\frac{s}{2}} \phi_{\varepsilon}(x) \mathrm{d} x \\
& -2 \int_{\mathbb{R}^{n}}(-\Delta)^{\frac{s}{2}} u_{n}(x) \int_{\mathbb{R}^{n}} \frac{\left(\phi_{\varepsilon}(x)-\phi_{\varepsilon}(y)\right)\left(u_{n}(x)-u_{n}(y)\right)}{|x-y|^{n+s}} \mathrm{~d} x \mathrm{~d} y \\
& =\lim _{\varepsilon \rightarrow 0} \lambda \int_{B_{2 \varepsilon}\left(x_{k_{0}}\right)} V(x)\left|u_{n}\right|^{p}(x) \phi_{\varepsilon}(x) \mathrm{d} x+\int_{B_{2 \varepsilon}\left(x_{k_{0}}\right)} \phi_{\varepsilon}(x) \mathrm{d} v \\
& -\beta \int_{B_{2 \varepsilon}\left(x_{k_{0}}\right)} K(x) \phi_{\varepsilon}(x) \mathrm{d} \mu .
\end{aligned}
$$

Since $\phi$ is regular function with compact support, it is easy to see that it satisfies the hypothesis of Lemma 2.1, by Lemma 2.2 and Lemma 2.3 applied to the sequence $\left\{u_{n}\right\}$, it follows that the left hand side of (3.13) goes to zero. We obtain that$$
\lim _{\varepsilon \rightarrow 0}\left(\lambda \int_{B_{2 \varepsilon}\left(x_{k_{0}}\right)} V(x)\left|u_{n}\right|^{p}(x) \phi_{\varepsilon}(x) \mathrm{d} x+\int_{B_{2 \varepsilon}\left(x_{k_{0}}\right)} \phi_{\varepsilon}(x) \mathrm{d} v-\beta \int_{B_{2 \varepsilon}\left(x_{k_{0}}\right)} K(x) \phi_{\varepsilon}(x)\right) \mathrm{d} \mu
$$$$
=\beta K\left(x_{k_{0}}\right) v_{k_{0}}-\mu_{k_{0}}=0 \text {. }
$$

Clearly, if $K(x) \leq 0$, we get $\mu_{k_{0}}=v_{k_{0}}=0$; if $K\left(x_{k_{0}}\right)>0$, by (3.8), we get $v_{k_{0}}=0 \quad$ or $\quad v_{k_{0}} \geq\left[\frac{S(n, s)}{\beta K\left(x_{k_{0}}\right)}\right]^{\frac{n}{2 s}}$.

suppose that $v_{k_{0}} \neq 0$, we know that

$$
0>c=\lim _{n \rightarrow \infty}\left[J\left(u_{n}\right)-\frac{1}{2_{s}^{*}}\left\langle J^{\prime}\left(u_{n}\right), u_{n}\right\rangle\right]
$$

according to the embedded theorem, we have

$$
\begin{aligned}
0>c & \geq\left(\frac{1}{2}-\frac{1}{2_{s}^{*}}\right)\left\|u_{n}\right\|_{X_{0}^{s}(\Omega)}^{2}-\left(\frac{\lambda}{p}-\frac{\lambda}{2_{s}^{*}}\right) \int_{\Omega} V(x)\left|u_{n}\right|^{p} \mathrm{~d} x \\
& =\frac{s}{n}\left\|u_{n}\right\|_{X_{0}^{s}(\Omega)}^{2}-\left(\frac{\lambda}{p}-\frac{\lambda}{2_{s}^{*}}\right) \int_{\Omega} V(x)\left|u_{n}\right|^{p} \mathrm{~d} x \\
& \geq \frac{s}{n} S^{-1}(n, s)\left\|u_{n}\right\|_{L^{2_{s}^{*}(\Omega)}}^{2}-\left(\frac{\lambda}{p}-\frac{\lambda}{2_{s}^{*}}\right) S^{-\frac{p}{2}}(n, s)\|V(x)\|_{L^{q}(\Omega)}\left\|u_{n}\right\|_{L^{*} s}^{p} .
\end{aligned}
$$


This yields that

$$
\begin{aligned}
& \|u\|_{L^{2_{s}^{*}(\Omega)}} \leq C \lambda^{\frac{1}{2-p}} . \\
& \text { Thus, if } v_{k_{0}} \geq\left[\frac{S(n, s)}{\beta K\left(x_{k_{0}}\right)}\right]^{\frac{n}{2 s}} \text {, we get that } \\
& 0>c=\lim _{n \rightarrow \infty}\left[J\left(u_{n}\right)-\frac{1}{2_{s}^{*}}\left\langle J^{\prime}\left(u_{n}\right), u_{n}\right\rangle\right] \\
& \geq\left(\frac{1}{2}-\frac{1}{2_{s}^{*}}\right)\|u\|_{X_{0}^{s}(\Omega)}^{2}+\frac{s}{n} \mu_{k_{0}}-\left(\frac{\lambda}{p}-\frac{\lambda}{2_{s}^{*}}\right) \int_{\Omega} V(x)|u|^{p} \mathrm{~d} x \\
& \geq \frac{s}{n} S^{-1}(n, s)\|u\|_{L^{*} s(\Omega)}^{2}+\frac{s}{n} \mu_{k_{0}}-\left(\frac{\lambda}{p}-\frac{\lambda}{2_{s}^{*}}\right) S^{-\frac{p}{2}}(n, s)\|V(x)\|_{L^{q}(\Omega)}\|u\|_{L^{2_{s}^{*}}}^{p} \\
& \geq \frac{s}{n} S^{-1}(n, s)\|u\|_{L^{*} s_{(\Omega)}^{*}}^{2}+\frac{s}{n} \mu_{k_{0}}-\left(\frac{\lambda}{p}-\frac{\lambda}{2_{s}^{*}}\right) S^{-\frac{p}{2}}(n, s)\|V(x)\|_{L^{q}(\Omega)}\|u\|_{L^{*} s}^{p} \\
& \geq \frac{s}{n} S(n, s) v_{k_{0}}^{-\frac{2_{s}^{*}}{2}}-\left(\frac{\lambda}{p}-\frac{\lambda}{2_{s}^{*}}\right) S^{-\frac{p}{2}}(n, s)\|V(x)\|_{L^{p}(\Omega)}\|u\|_{L^{2 *}}^{p} \\
& \geq \frac{s}{n} S^{\frac{n}{2 s}}(n, s)\left[\beta K\left(x_{k_{0}}\right)\right]^{\frac{2 s-n}{2 s}}-C \lambda^{\frac{2}{2-p}} \text {. }
\end{aligned}
$$

However, if $\beta>0$ is given, we can choose $\lambda_{0}>0$ so small for every $0<\lambda<\lambda_{0}$ that last term on the right-hand side above is greater than 0 which is contradiction when $2<p<2_{s}^{*}$

$$
\begin{aligned}
0 & >c=\lim _{n \rightarrow \infty}\left[J\left(u_{n}\right)-\frac{1}{p}\left\langle J^{\prime}\left(u_{n}\right), u_{n}\right\rangle\right] \\
& =\left(\frac{1}{2}-\frac{1}{p}\right)\|u\|_{X_{0}^{s}(\Omega)}^{2}-\left(\frac{\beta}{2_{s}^{*}}-\frac{\beta}{p}\right) \int_{\Omega} K(x)|u|^{2_{s}^{*}} \mathrm{~d} x \\
& \geq\left(\frac{1}{2}-\frac{1}{p}\right)\|u\|_{X_{0}^{s}(\Omega)}^{2}-\left(\frac{\beta}{2_{s}^{*}}-\frac{\beta}{p}\right) \int_{\Omega \cap\{K(x)<0\}} K(x)|u|^{2_{s}^{*}} \mathrm{~d} x \\
& \geq\left(\frac{1}{2}-\frac{1}{p}\right)\|u\|_{X_{0}^{s}(\Omega)}^{2}-\left(\frac{\beta}{2_{s}^{*}}-\frac{\beta}{p}\right)\|K(x)\|_{L^{\infty}}\|u\|_{L^{2} s}^{2_{s}^{*}}
\end{aligned}
$$

$\beta$ is the same as $\lambda$ greater than 0 . We see that $v_{k_{0}} \geq\left[\frac{S(n, s)}{\beta K\left(x_{k_{0}}\right)}\right]^{\frac{n}{2 s}}$ cannot occur if $\lambda_{0}$ or $\beta_{0}$ are choose properly. Thus $\mu_{k}=v_{k}=0$. As consequence, we obtain that $\left(u_{n}\right)_{+}-u \rightarrow 0$ in $L^{2_{s}^{*}}(\Omega)$, that is $\lim _{n \rightarrow \infty} \int_{R^{n}}\left|\left(u_{n}\right)_{+}\right|^{2_{s}^{*}} \mathrm{~d} x=\int_{R^{n}}|u|^{2_{s}^{*}} \mathrm{~d} x$. This implies convergence of $\lambda V(x)\left|u_{n}\right|^{p-1}+\beta K(x)\left|u_{n}\right|^{2_{s}^{*}-1}$ in $L^{2_{s}^{*}}(\Omega)$. Finally using the continuity of the inverse operator $(-\Delta)^{s}$. We obtain strong convergence of $u_{n}$ in $X_{0}^{s}(\Omega)$.\#

Next, by using the classical concept and properties of the genus, we construct a min-max class of the critical point. 
For a Banach space $X$, We define the set

$\mathcal{A}=\{A \subset X \backslash\{0\}: A$ is closed in $X$ and symmetric with respect to the orign $\}$

For $\mathcal{A} \in A$, define

$$
\gamma(A):=\inf \left\{m \in N, \exists \phi \in C\left(A, R^{m} \backslash\{0\}\right), \phi(x)=-\phi(-x)\right\}
$$

If there is no mapping $\phi$ as above for any $m \in N$, there $\gamma(A)=+\infty$. we refer to [8] for the properties of the genus.

Proposition 3.3. [8] Let $A, B \subset \mathrm{A}$,

1) If there exists an odd map $f \in C(A, B)$, then $\gamma(A) \leq \gamma(B)$;

2) If $A \subset B$, then $\gamma(A) \leq \gamma(B)$;

3) $\gamma(A \cup B) \leq \gamma(A)+\gamma(B)$;

4) If $S$ is a sphere centered at the origin in $R^{m}$, then $\gamma(s)=m$;

5) If $A$ is compact, there exists a symmetric Neighborhood $N$ of $A$, such that $\gamma(\bar{N})=\gamma(A)$.

According Holder inequality, we get that

$$
\begin{aligned}
J(u) & =\frac{1}{2}\|u\|_{X_{0}^{s}}^{2}-\frac{\lambda}{p} \int_{\Omega} V(x)|u|^{p} \mathrm{~d} x-\frac{\beta}{2_{s}^{*}} \int_{\Omega} K(x)|u|^{2_{s}^{*}} \mathrm{~d} x \\
& \geq \frac{1}{2}\|u\|_{X_{0}^{s}}^{2}-C_{1} \lambda\|u\|_{X_{0}^{s}}^{p}-C_{2} \beta\|u\|_{X_{0}^{s}}^{2_{s}^{*}}
\end{aligned}
$$

We define the function

$$
Q(t):=\frac{1}{2} t^{2}-C_{1} \lambda t^{p}-C_{2} \beta t^{2_{s}^{*}}
$$

Then it is easy to see that given $\beta>0$, there exists $\lambda_{1}>0$ so small that for every $0<\lambda<\lambda_{1}$, there exists $0<T_{0}<T_{1}$ such that $Q(t)<0$ for $0 \leq t \leq T_{0}$, $Q(t)>0$ for $T_{0}<t<T_{1}$. and $Q(t)<0 \quad t>T_{1}$. Analogously, for given $\lambda>0$, we can choose $\beta_{1}>0$ with the property that $T_{0}, T_{1}$ as above for each $0<\beta<\beta_{1}$. Clearly, $Q\left(T_{0}\right)=Q\left(T_{1}\right)=0$.

As in [9], Let $\tau: \mathbb{R}^{+} \rightarrow[0,1]$ be a nonincreasing $C^{\infty}$ function such that $\tau(t)=1$ if $0 \leq \tau \leq T_{0}$ and $\tau(t)=0$. if $\tau \geq T_{0}$. Set $\Psi(u)=\tau\left(\|u\|_{X_{0}^{s}(\Omega)}\right)$, we make the following truncation of the function $J$ :

$$
\tilde{J}(u)=\frac{1}{2}\|u\|_{X_{0}^{s}}^{2}-\frac{\lambda}{p} \int_{\Omega} V(x)|u|^{p} \mathrm{~d} x-\frac{\beta}{2_{s}^{*}} \psi(u) \int_{\Omega} K(x)|u|^{2_{s}^{*}} \mathrm{~d} x
$$

then

$$
\tilde{J}(u) \geq \tilde{Q}\|u\|_{X_{0}^{s}(\Omega)} .
$$

where $\tilde{Q}(t):=\frac{1}{2} t^{2}-C_{1} \lambda t^{p}-C_{2} \beta t^{2_{s}^{*}} \psi(t)$.

It is clear that $\tilde{J}(u) \in C^{1}$ and is bounded from below.

Lemma 3.4. [10] 1) For any $\lambda>0$ and $0<\beta<\beta_{1}$ or any $\beta>0$ and $0<\lambda<\lambda_{1}$, if $\tilde{J}(u)<0$, then $\|u\|_{X_{0}^{s}(\Omega)}<T_{0}$ and $\tilde{J}(u)=J(u)$.

2) For any $\lambda>0$, there exists such that if $0<\beta<\bar{\beta}$ and $c<0$ then $\tilde{J}$ satisfies $(P S)_{c}$. 
3) For any $\beta>0$, there exists $\tilde{\lambda}>0\left(\tilde{\lambda} \leq \lambda_{1}\right)$ such that if $0<\lambda<\tilde{\lambda}$ and $c<0$ then $\tilde{J}$ satisfies $(P S)_{c}$.

Lemma 3.5. Denote $\tilde{J}^{\alpha}:=\left\{u \in X_{0}^{s}(\Omega), \tilde{J}(u) \leq \alpha\right\}$. Then for any $m \in N$, there is $\varepsilon_{m}<0$ such that $\gamma\left(\tilde{J}^{\varepsilon_{m}}\right) \geq m$.

Proof. Denote by $X_{0}^{s}(\Omega)$ the closure of $C_{0}^{\infty}(\Omega)$ with the respect to norm $\|u\|_{X_{0}^{s}(\Omega)}=\left(\int_{\Omega} \frac{|u(x)-u(y)|^{2}}{|x-y|^{n+2 s}} \mathrm{~d} x \mathrm{~d} y\right)^{\frac{1}{2}}, V(x)>0$ in $\Omega$. Extending functions in $X_{0}^{s}(\Omega)$ by 0 outside $\Omega$. Let $X_{m}$ be a m-dimensional subspace of $X_{0}^{s}(\Omega)$. For any $u \in X_{m}, u \neq 0$. We write $u=r_{m} w$ with $w \in X_{m}$ and $\|w\|_{X_{0}^{s}(\Omega)}=1$. From the assumptions of $V(x)$, it is easy to see for every $w \in X_{m}$ with $\|w\|_{X_{0}^{s}(\Omega)}=1$ that there exists $d_{m}>0$ such that

$$
\int_{\Omega} V(x)|w|^{p} \mathrm{~d} x \geq d_{m}
$$

For $0<r_{m}<T_{0}$. Since all the norms are equivalent, we get

$$
\begin{aligned}
\tilde{J}(u)=J(u) & =\frac{1}{2}\|u\|_{X_{0}^{s}(\Omega)}^{2}-\frac{\lambda}{p} \int_{\Omega} V(x)|u|^{p} \mathrm{~d} x-\frac{\beta}{2_{s}^{*}} \int_{\Omega} K(x)|u|^{2_{s}^{*}} \mathrm{~d} x \\
& \leq \frac{1}{2}\|u\|_{X_{0}^{s}(\Omega)}^{2}-\frac{\lambda}{p} \int_{\Omega} V(x)|u|^{p} \mathrm{~d} x+\left.\frac{\beta}{2_{s}^{*}}\left|\int_{\Omega} K(x)\right| u\right|^{2_{s}^{*}} \mathrm{~d} x \mid \\
& \leq \frac{1}{2} r_{m}^{2}-\lambda c d_{m}+c \beta r_{m}^{2_{s}^{*}}:=\varepsilon_{m} .
\end{aligned}
$$

Therefore for given $\lambda$ and $\beta$. we can choose $r_{m} \in\left(0, T_{0}\right)$ sufficiently small so that $\tilde{J}(u) \leq \varepsilon_{m}<0$. \#

Let $S_{r_{m}}=\left\{u \in X_{0}^{s}(\Omega):\|u\|_{X_{0}^{s}(\Omega)}=r_{m}\right\}$. Then $S_{r_{m}} \cap X_{m} \subset \tilde{J}^{\varepsilon_{m}}$, Hence by proposition 3.3 (2) and (4) $r\left(\tilde{J}^{\varepsilon_{m}}\right) \geq r\left(S_{r_{m}} \cap X_{m}\right) \geq m$.

We denote $\Gamma_{m}=\{A \in \mathrm{A}: \gamma(A) \geq m\}$ and let

$$
C_{m}:=\inf _{A \in \Gamma_{m}} \sup _{u \in A} J(u)
$$

then

$$
-\infty<C_{m} \leq \varepsilon_{m}<0, \quad m \in N
$$

because $\tilde{J}^{\varepsilon_{m}} \in \Gamma_{m}$ and $\tilde{J}$ is bounded from below.

Proposition 3.6. Let $\lambda, \beta$ be as in Lemma 3.5 (2) and (3). Then all $c_{m}$ given by (3.25) are critical values of $\tilde{J}$ and $c_{m} \rightarrow 0$ as $m \rightarrow 0$.

Proof. Denote $K_{\varepsilon}=\left\{u \in X_{0}^{s}(\Omega): \tilde{J}(u)=c, \tilde{J}^{\prime}(u)=0\right\}$. Then by Lemma 3.4 (2) and (3), if $c<0, K_{c}$ is compact. It is clear that $C_{m} \leq C_{m+1}$. By (3.26) $C_{m}<0$. Hence $C_{m} \rightarrow \bar{C} \leq 0$. Moreover, since $(P S)_{c}$ satisfied, it follows from a standard argument (see [11]) that all $C_{m}$ are critical values of $\tilde{J}$. Now, we claim that $\bar{c}=0$. If $\bar{c}<0$ because $K_{\bar{c}}$ is compact and $K_{\bar{c}} \in A$, it follows from Proposition 3.3 (5) that $\gamma\left(K_{\bar{c}}\right)=m_{0}<+\infty$ and there exists $\delta>0$ such that $\gamma\left(K_{\bar{c}}\right)=\gamma\left(N_{\delta}\left(K_{\bar{c}}\right)\right)=m_{0}$. By the deformation Lemma [9], there exists $\varepsilon>0(\bar{c}+\varepsilon<0)$ and an odd homeomorphism $\varsigma(\cdot): X_{0}^{s}(\Omega) \rightarrow X_{0}^{s}(\Omega)$ such 
that

$$
\varsigma\left(\tilde{J}^{\bar{c}+\varepsilon} \backslash N_{\delta}\left(K_{\bar{c}}\right)\right) \subset \tilde{J}^{\bar{c}-\varepsilon}
$$

Since $c_{m}$ is increasing anad converges to $\bar{c}$. there exists $m \in N$ such that

$$
c_{m}>\bar{c}-\varepsilon .
$$

And exists a $A \in \Gamma_{m+m_{0}}$ such that

$$
\sup _{u \in A} \tilde{J}(u)<\bar{c}+\varepsilon
$$

By Proposition 3.3 (3), we obtain

$$
\gamma\left(\overline{A \backslash N_{\delta}\left(K_{\bar{c}}\right)}\right) \geq \gamma(A)-\gamma\left(N_{\delta}\left(K_{\bar{c}}\right)\right) \geq m
$$

By Proposition 3.3 (1), we obtain

$$
\gamma\left(\overline{\varsigma\left(A \backslash N_{\delta}\left(K_{\bar{c}}\right)\right)}\right) \geq m
$$

therefore

$$
\varsigma\left(A \backslash N_{\delta}\left(K_{\bar{c}}\right)\right) \in \Gamma_{m}
$$

consequently, from (3.28), we get

$$
\sup _{u \in \zeta\left(A \backslash N_{\delta}\left(K_{\bar{c}}\right)\right)} \tilde{J}(u) \geq c_{m}>\bar{c}-\varepsilon
$$

on the other hand, by (3.27) and (3.29)

$$
\varsigma\left(A \backslash N_{\delta}\left(K_{\bar{c}}\right)\right) \subset \varsigma\left(\tilde{J}^{\bar{c}+\varepsilon} \backslash N_{\delta}\left(K_{\bar{c}}\right)\right) \subset \tilde{J}^{\bar{c}-\varepsilon}
$$

which implies that

$$
\sup _{u \in \varsigma\left(A \backslash N_{\delta}\left(K_{\bar{c}}\right)\right)} \tilde{J}(u) \leq \bar{c}-\varepsilon
$$

this contradicts to (3.32). Hence $c_{m} \rightarrow 0$.\#

By (1) of Lemma $3.4 \tilde{J}(u)=J(u)$ if $\tilde{J}(u)<0$. This and Proposition 3.6 give Theorem1.1.

\section{Conflicts of Interest}

The author declares no conflicts of interest regarding the publication of this paper.

\section{References}

[1] Lions, P.-L. (1985) The Concentration-Compactness Principle in the Calculus of Variations. The Limit Case. II. Revista Matemática Iberoamericana, 1, 45-121.

[2] Nezza, E.D., Palatucci, G. and Valdinoci, E. (2012) Hitchhiker's Guide to the Fractional Sobolev Spaces. Bulletin Des Sciences Mathématiques, 136, 521-573. https://doi.org/10.1016/j.bulsci.2011.12.004

[3] Adams, R. (1975) Sobolev Spaces. Academic Press.

[4] Barrios, B., Colorado, E. and Servadei, R. (2015) A Critical Fractional Equation with Concave-Convex Power Nonlinearities. Annales De Linstitut Henri Poincaré, 32, 
875-900. https://doi.org/10.1016/j.anihpc.2014.04.003

[5] Xuan, B.J. (2006) Theory and Application of Variational Method. China University of Science and Technology Press, Anhui.

[6] Meyers, N. and Serrin, J. (1964) H = W. Proceedings of the National Academy of Sciences of the USA, 51, 1055-1056. https://doi.org/10.1073/pnas.51.6.1055

[7] Palatucci, A. and Pisante, G. (2014) Improved Sobolev Embeddings, Profile Decomposition and Concentration-Compactness for Fractional Sobolev Spaces. Calculus of Variations and Partial Differential Equations, 50, 799-829.

[8] Ambrosetti, A. and Rabinowitz, P.H. (1973) Dual Variational Methods in Critical Point Theory and Applications. Journal of Functional Analysis, 14, 349-381.

https://doi.org/10.1016/0022-1236(73)90051-7

[9] Azorero, J.G. and Alonso, I.P. (1991) Multiplicity of Solutions for Elliptic Problems with Critical Exponent or with a Nonsymmetric Term. Transactions of the American Mathematical Society, 323, 877-895. https://doi.org/10.2307/2001562

[10] Youjun, W. (2018) Multiplicity of Solutions for Singular Quasilinear Schrödinger Equations with Critical Exponents. Journal of Mathematical Analysis and Applications, 458, 1027-1043.

[11] Rabinowitz, P. (1986) Minimax Methods in Critical Points Theory with Applications to Differential Equation. $C B M S, 65$, AMS, Providence.

https://doi.org/10.1090/cbms/065 